CORRECTION

https://doi.org/10.1038/s41586-018-0248-2

\title{
Publisher Correction: Observation of anisotropic magneto-Peltier effect in nickel
}

Ken-ichi Uchida, Shunsuke Daimon, Ryo Iguchi \& Eiji Saitoh

Correction to: Nature https://doi.org/10.1038/s41586-018-0143-x, published online 21 May 2018.

In this Letter, owing to an error during the production process, ' $\theta_{H}$ ' was incorrectly written as ' $\theta_{\mathrm{HH}}$ ' six times in the paragraph starting "Up to now,...”. These errors have been corrected online. 